\title{
Finite Element Methods of Optimal Order for Problems with Singular Data
}

\author{
By Kenneth Eriksson
}

\begin{abstract}
An adapted finite element method is proposed for a class of elliptic problems with singular data. The idea is to subtract the main singularity from the solution and to solve for the remainder using suitable mesh-refinements. Optimal order error estimates are proved.
\end{abstract}

1. Introduction and Results. Let $\Omega$ be a bounded domain in $R^{N}$ with smooth boundary $\Gamma$ and consider the following problem: Given $x_{0} \in \Omega$ find $u=u(x)$ such that

$$
\begin{aligned}
L u(x) \equiv & -\sum_{i, j=1}^{N} \frac{\partial}{\partial x_{j}}\left(a_{i j}(x) \frac{\partial u}{\partial x_{i}}\right)+\sum_{i=1}^{N} a_{i}(x) \frac{\partial u}{\partial x_{i}}+a(x) u \\
& =\delta\left(x-x_{0}\right) \quad \text { in } \Omega, \\
& l u(x) \equiv \sum_{i, j=1}^{N} a_{i j}(x) \frac{\partial u}{\partial x_{i}} n_{j}(x)=0 \text { on } \Gamma,
\end{aligned}
$$

where $\delta$ is the Dirac distribution (unit impulse), $n=\left(n_{j}\right)$ is the outward unit normal to $\Gamma$, and $a_{i j}, a_{i}$, and $a$ are smooth ( $C^{\infty}$ regular) functions on $\bar{\Omega}$, with $a_{i j}=a_{j i}$, and such that the associated bilinear form

$$
A(v, w) \equiv \int_{\Omega}\left(\sum_{i, j} a_{i j} \frac{\partial v}{\partial x_{i}} \frac{\partial w}{\partial x_{j}}+\sum_{i} a_{i} \frac{\partial v}{\partial x_{i}} w+a v w\right) d x
$$

satisfies the ellipticity-coercivity condition

$$
A(v, v) \geqslant c\|v\|_{1, \Omega}^{2} \quad \text { for all } v \in H^{1}(\Omega),
$$

where $c$ is a positive constant and $\|\cdot\|_{1, \Omega}$ the usual norm in $H^{1}(\Omega)$, the space of functions with square-integrable first-order derivatives in $\Omega$. It is well known (cf., e.g., [8]) that problem (1.1) admits a unique (distributional) solution $u$, which is also determined by the corresponding variational equations

$$
A(u, \psi)=\psi\left(x_{0}\right) \text { for all } \psi \in W_{\infty}^{1}(\Omega),
$$

where $W_{\infty}^{1}(\Omega)$ is the space of functions with bounded first-order derivatives on $\bar{\Omega}$.

Received January 10, 1983; revised July 27, 1984.

1980 Mathematics Subject Classification. Primary 65N15, 65N30.

Key words and phrases. Neumann problem, Green's function, adapted finite element methods, mesh-refinement, optimal order, error estimate. 
In general, problem (1.1) (or (1.3)) cannot be solved exactly, so we are faced with the problem of finding an accurate approximate solution. We shall consider the following standard approach for this (cf., e.g., [1], [7], [11]): Given a finite-dimensional space $S_{h} \subset H^{1}(\Omega) \cap C(\bar{\Omega})$ find $u_{h} \in S_{h}$ such that

$$
A\left(u_{h}, \chi\right)=\chi\left(x_{0}\right) \text { for all } \chi \in S_{h} .
$$

By the coercivity of $A(\cdot, \cdot)$, there exists a unique such $u_{h}$ determined by the linear system of equations

$$
\sum_{i=1}^{M} U_{i} A\left(\chi_{i}, \chi_{j}\right)=\chi_{j}\left(x_{0}\right) \text { for } j=1, \ldots, M,
$$

where $u_{h}=\sum_{i=1}^{M} U_{i} \chi_{i}$, and $\left\{\chi_{j}\right\}_{j=1}^{M}$ is an arbitrary basis for $S_{h}$. For appropriate finite element spaces $S_{h}$, where $h$ is the associated mesh-size parameter, there exist a priori estimates for the error $u_{h}-u$ in terms of $h$. One problem in deriving these estimates is that the singularity of $u$ at $x_{0}$, which is of order $\log \left|x-x_{0}\right|^{-1}$ for $N=2$, and $\left|x-x_{0}\right|^{-N+2}$ for $N \neq 2$, frustrates the usual type of error analysis. For $N=1$ this is a minor problem since then the singularity is concentrated at $x_{0}$ and $u$ is continuous. Choosing $x_{0}$ as one of the nodal points and using continuous piecewise polynomials for $S_{h}$, the usual analysis carries through, and, for example, for polynomials of degree $r-1$ we have $\left\|u_{h}-u\right\|_{1, \Omega} \leqslant C h^{r-1}$. For $N>1$, however, the standard method of analysis fails since then the solution does not even belong to $H^{1}(\Omega)$. Nevertheless, Babuška [1] was able to show, for $N=2, L=-\Delta+I$ (minus Laplacian plus identity), and finite element spaces $S_{h}$ possessing standard approximation and inverse properties (such as piecewise linears on a quasi-uniform triangulation of $\Omega$ ), that

$$
\left\|u_{h}-u\right\|_{0, \Omega} \leqslant C_{\varepsilon} h^{1-\varepsilon},
$$

where $\|\cdot\|_{0, \Omega}$ is the $L_{2}(\Omega)$-norm and $\varepsilon>0$ is arbitrary. Later, Scott [11] improved and generalized this result by showing that for elliptic operators of order $2 m$, normal covering boundary conditions (cf., e.g., [9]), and dimension $N \geqslant 2$, one has

$$
\left\|u_{h}-u\right\|_{s, \Omega} \leqslant C\left(x_{0}\right) h^{2 m-s-N / 2} \text { for } 2 m-r \leqslant s<2 m-N / 2,
$$

where $C\left(x_{0}\right)$ tends to infinity as $x_{0}$ approaches $\Gamma, r \geqslant 2 m$ is the order of approximation of $S_{h} \subset H^{s}(\Omega)$, and the Sobolev norm index $s$ may also be negative (cf., e.g., [9] for the definition). Despite its generality, Scott's result falls short in a certain respect. For instance, if $m=1, r=2$ (piecewise linears), and $N=2$ or 3 , we obtain no information about $\nabla\left(u_{h}-u\right)$, and for $N \geqslant 4$, no information whatsoever.

Recently, in [7], we proposed the use of adapted finite element spaces for the given problem. Denoted by $S_{h}\left(x_{0}, \alpha, r\right)$ where $h \in\left(0, \frac{1}{2}\right], \alpha \in[0,1)$, and $r \geqslant 2$ are parameters, these spaces can be described as follows: Given positive constants $c$ and $C$ let $\Omega$ be divided into elements $\tau$ such that

$$
c \operatorname{diam}(\tau) \leqslant h\left(\operatorname{dist}\left(x_{0}, \tau\right)\right)^{\alpha}+h^{1 /(1-\alpha)} \leqslant C \operatorname{diam}(\tau) \text { for all } \tau,
$$

where each $\tau$ is the restriction to $\Omega$ of the interior of a $N$-simplex $\hat{\tau}$ (cf. [4]), with

$$
(\operatorname{diam}(\hat{\tau}))^{N} \leqslant C \int_{\tau} d x
$$


and the intersection of any two such simplices is either a face of both, or of lower dimension. For $S_{h}=S_{h}\left(x_{0}, \alpha, r\right)$ we take the space of all continuous functions on $\bar{\Omega}$ which reduces to polynomials of degree at most $r-1$ on each $\tau \subset \Omega$. By (1.5) the mesh is refined (graded) around $x_{0}$ in such a way that elements at distance $d$ from $x_{0}$ have diameters of order $h d^{\alpha}$, but a minimum diameter of order $h^{1 /(1-\alpha)}$. Hence $h$ is a parameter for the maximal global mesh-size, and $\alpha$ determines the degree of refinement. Of course, such a mesh-refinement enables a better approximation in $S_{h}$ of any given function which is irregular near $x_{0}$. The condition (1.6) is used to derive local inverse estimates.

The following results were obtained in [7] for $u_{h} \in S_{h}\left(x_{0}, \alpha, r\right)$ being the solution of (1.4). For $\alpha>(r-2) /(r-1)$,

$$
\left\|\nabla\left(u_{h}-u\right)\right\|_{L_{1}(\Omega)} \leqslant C h^{r-1},
$$

and for $\alpha>(r-2) / r$,

$$
\left\|u_{h}-u\right\|_{L_{1}(\Omega)} \leqslant C h^{r},
$$

where $C$ is a constant independent of $h$ and $x_{0}$. Further, if $\alpha>(r-1) / r$, and if $d \equiv\left|x-x_{0}\right| \geqslant c h^{1 /(1-\alpha)}$ and $\operatorname{dist}(x, \Gamma) \geqslant d$ for a suitable $c>0$, then

$$
\left|u_{h}(x)-u(x)\right| \leqslant C h^{r}(\ln 1 / h)^{\bar{r}} d^{-N},
$$

where $\bar{r}=1$ if $r=2, \bar{r}=0$ if $r>2$.

In this paper, we analyze a method to approximate the solution of (1.1) to the accuracy (1.7), (1.8), and (1.9) which requires a lesser degree of mesh-refinement than the one in [7]. One reason for introducing such a method is that the computational effects of strong mesh-refinements are not yet very well known. Recall that the condition number for the stiffness matrix $\left(A\left(\chi_{i}, \chi_{j}\right)\right)$ depends on the mesh-size.

In order to describe the method we first note that the solution of (1.1) can be written in the form

$$
u=u_{0}+v
$$

where $u_{0}$ is the fundamental singularity of $u$ defined by (in the sequel we only consider the case $N \geqslant 2$ )

$$
u_{0}(x)= \begin{cases}\frac{|\operatorname{det}(Q)|}{2 \pi} \ln \left(\left|\hat{x}-\hat{x}_{0}\right|^{-1}\right) & \text { if } N=2, \\ \frac{|\operatorname{det}(Q)|}{(N-2) \sigma_{N}}\left|\hat{x}-\hat{x}_{0}\right|^{-N+2} & \text { if } N>2,\end{cases}
$$

where $Q$ is the inverse of $A^{1 / 2}$, the positive square root of $A \equiv\left(a_{i j}\left(x_{0}\right)\right), \hat{x}-\hat{x}_{0}=$ $Q\left(x-x_{0}\right)$, and $\sigma_{N}$ is the surface area of the unit ball $B_{1}(0) \subset R^{N}$. For example, if $L=-\Delta+I$ we can take $Q=I$ and thus obtain

$$
u_{0}(x)=\frac{1}{(N-2) \sigma_{N}}\left|x-x_{0}\right|^{-N+2} \quad(N>2),
$$

which we recognize as the fundamental solution of $-\Delta$. It is a matter of straightforward calculation to verify that in the general case $u_{0}$ satisfies

$$
-\sum_{i, j=1}^{N} a_{i j}\left(x_{0}\right) \frac{\partial^{2} u_{0}}{\partial x_{j} \partial x_{i}}=\delta\left(x-x_{0}\right) \text { in } \Omega \text {. }
$$


In view of (1.10) we are led to seek an approximate solution of (1.1) in the form

$$
\tilde{u}_{h}=u_{0}+v_{h}, \quad \text { with } v_{h} \in S_{h}=S_{h}\left(x_{0}, \alpha, r\right) \text {, }
$$

and such that

$$
A\left(\tilde{u}_{h}, \chi\right)=\chi\left(x_{0}\right) \text { for all } \chi \in S_{h} .
$$

Again, by the coercivity of $A(\cdot, \cdot)$, there is a unique such $\tilde{u}_{h}$. In fact, to seek $\tilde{u}_{h}$ is to seek $v_{h}=\sum_{i=1}^{M} V_{i} \chi_{i} \in S_{h}$ such that

$$
\sum_{i=1}^{M} V_{i} A\left(\chi_{i}, \chi_{j}\right)=\chi_{j}\left(x_{0}\right)-A\left(u_{0}, \chi_{j}\right) \text { for } j=1, \ldots, M,
$$

where $\left\{\chi_{j}\right\}_{j=1}^{M}$ is a basis for $S_{h}$.

We shall prove the following error estimates for this method:

THEOREM 1. Let $u$ be the solution of (1.1) and $\tilde{u}_{h}=u_{0}+v_{h}$ that of (1.14), with $v_{h} \in S_{h}\left(x_{0}, \alpha, r\right)$. Then, for $\alpha>(r-3) /(r-1)$,

$$
\left\|\nabla \tilde{u}_{h}-\nabla u\right\|_{L_{1}(\Omega)} \leqslant C h^{r-1},
$$

and for $\alpha>(r-3) / r$,

$$
\left\|\tilde{u}_{h}-u\right\|_{L_{1}(\Omega)} \leqslant C h^{r}
$$

where $C$ may depend on the given problem as well as on $\alpha, r$, and the constants in (1.5) and (1.6), but not on $h$.

Further, we have the following pointwise error estimate:

THEOREM 2. Let $u$ and $\tilde{u}_{h}$ be as in Theorem 1 with $\alpha>(r-3) / r$. Then

$$
\left|\tilde{u}_{h}(x)-u(x)\right| \leqslant C h^{r}(\ln 1 / h)^{\bar{r}}\left|x-x_{0}\right|^{-N} \text { for } x \in \bar{\Omega}, x \neq x_{0},
$$

where $C$ is independent of $x$ and $h$, and $\bar{r}=1$ if $r=2, \bar{r}=0$ if $r>2$.

Remark. The constants $C$ in Theorems 1 and 2 become infinite as $x_{0}$ approaches $\Gamma$. In order to have a method which is effective also when $x_{0}$ is close to (or even on) the boundary $\Gamma$ one can modify the definition of $u_{0}$ according to

$$
u_{0}(x)= \begin{cases}\frac{|\operatorname{det}(Q)|}{2 \pi}\left(\ln \left(\left|\hat{x}-\hat{x}_{0}\right|^{-1}\right)+\ln \left(\left|\hat{x}-\hat{x}_{0}^{*}\right|^{-1}\right)\right) & \text { if } N=2, \\ \frac{|\operatorname{det}(Q)|}{(N-2) \sigma_{N}}\left(\left|\hat{x}-\hat{x}_{0}\right|^{-N+2}+\left|\hat{x}-\hat{x}_{0}^{*}\right|^{-N+2}\right) & \text { if } N>2,\end{cases}
$$

where $\hat{x}_{0}^{*}$ is the " $Q$-reflexion" of $\hat{x}_{0}$ in $\Gamma$ defined by $\hat{x}_{0}^{*}=Q x_{0}^{*}, x_{0}^{*}=2 z-x_{0}$, and $z$ minimizes $\left|Q\left(y-x_{0}\right)\right|, y \in \Gamma$. For this modification of the method the estimates of Theorems 1 and 2 hold with constants $C$ independent of $x_{0}$.

The proofs of Theorems 1 and 2 are given in Sections 4 and 5, respectively. Sections 2 and 3 are devoted to preparatory work.

2. Preliminaries. Throughout this paper we shall denote by $c$ and $C$ various positive constants which are independent of $h$ (but which may depend on the data of the given problem (1.1), the constants in (1.5) and (1.6), and on the parameters $\alpha$ and $r$ ). Similarly, $C_{1}$ and $C_{*}$ will denote two specific such constants. 
Besides the usual $L_{p}$-norms

$$
\|v\|_{L_{p}\left(\Omega^{\prime}\right)}=\left(\int_{\Omega^{\prime}}|v(x)|^{p} d x\right)^{1 / p} \quad \text { for } p=1 \text { and } p=2,
$$

and

$$
\|v\|_{L_{\infty}\left(\Omega^{\prime}\right)}=\underset{x \in \Omega^{\prime}}{\operatorname{ess} \sup }|v(x)|
$$

we shall use the Sobolev norms

$$
\|v\|_{k, \Omega^{\prime}}=\left(\sum_{|\beta| \leqslant k}\left\|D^{\beta} v\right\|_{L_{2}\left(\Omega^{\prime}\right)}^{2}\right)^{1 / 2},
$$

where $|\beta|=\beta_{1}+\cdots+\beta_{N}$ is the length of the multi-index $\beta=\left(\beta_{1}, \ldots, \beta_{N}\right)$, and

$$
D^{\beta}=\left(\frac{\partial}{\partial x_{1}}\right)^{\beta_{1}} \cdots\left(\frac{\partial}{\partial x_{N}}\right)^{\beta_{N}}
$$

In particular, $\|\cdot\|_{0, \Omega^{\prime}}$ denotes the usual $L_{2}\left(\Omega^{\prime}\right)$-norm. The Sobolev space $H^{k}\left(\Omega^{\prime}\right)$ is the space of all functions $w$ such that $\|w\|_{k, \Omega^{\prime}}$ is finite.

In the proofs we shall consider subdomains of $\Omega$ defined by

$$
D_{j} \equiv\left\{x \in \Omega: 2^{-(j+1)}<\left|x-x_{0}\right|<2^{-j}\right\}
$$

and

$$
\Omega_{j} \equiv\left\{x \in \Omega:\left|x-x_{0}\right|<2^{-j}\right\} \quad \text { for } j \text { integer, }
$$

and set $d_{j} \equiv 2^{-j}$ and $h_{j} \equiv h d_{j}^{\alpha}$. Accordingly, $d_{j}$ is proportional to the diameter of $D_{j}$ and $\Omega_{j}$, and as long as $j$ is not too large so that $h_{j}$ is smaller than the minimal mesh-size $h^{1 /(1-\alpha)}, h_{j}$ is proportional to the maximal mesh-size on $D_{j}$ and $\Omega_{j}$. We shall frequently use the obvious facts that $d_{j} \leqslant C d_{j+1}$ and $h_{j} \leqslant C h_{j+1}$, and we also note that since $\Omega$ is bounded, there is an integer $j_{1}$ such that $D_{j}$ is empty for $j<j_{1}$.

Due to the variable mesh-size a typical interpolant in $S_{h}\left(x_{0}, \alpha, r\right)$ approximates a given function with variable degree of accuracy over $\Omega$. The following three results are quoted from [7].

LEMMA 1. Given $w$ there is an interpolant $w_{I} \in S_{h}\left(x_{0}, \alpha, r\right)$ of $w$ such that for $j \leqslant J_{1}$,

$$
\left\|w-w_{I}\right\|_{1, \Omega_{j}} \leqslant C h_{j}\|w\|_{2, \Omega_{j-1}},
$$

and

$$
\left\|w-w_{I}\right\|_{1, D_{j}} \leqslant C h_{j}^{m-1}\|w\|_{m, D_{j}^{1}} \text { for } 2 \leqslant m \leqslant r,
$$

where $J_{1}$ is determined by $2^{-J_{1}}=C_{1} h^{1 /(1-\alpha)}$ for a suitable sufficiently large constant $C_{1}$, and $D_{j}^{1} \equiv \Omega_{j-1} \backslash \bar{\Omega}_{j+2}$.

The next lemma shows a similar property for the Galerkin approximation.

LEMMA 2. If $j \leqslant J_{1}$, and if $v_{h} \in S_{h}\left(x_{0}, \alpha, r\right)$ and $v$ satisfy

$$
A\left(v_{h}-v, \chi\right)=0 \text { for all } \chi \in S_{h}\left(x_{0}, \alpha, r\right) \text { with support in } \overline{D_{j}^{1}} \text {, }
$$

then

$$
\left\|v_{h}-v\right\|_{1, D_{j}} \leqslant C h_{j}^{r-1}\|v\|_{r, D_{j}^{1}}+C d_{j}^{-1}\left\|v_{h}-v\right\|_{0, D_{j}^{1}}
$$


From (1.6) we have the following inverse property:

Lemma 3. For any $\tau$ as in (1.6) and any polynomial $p$ of degree at most $r-1$, we have

$$
\|p\|_{L_{\infty}(\tau)} \leqslant C(\operatorname{diam}(\tau))^{-N / q}\|p\|_{L_{q}(\tau)} \text { for } q \in[1, \infty)
$$

For the proofs of these results we refer to [7].

3. A Step of Reduction. Recall that by definition $u=u_{0}+v$ and $\tilde{u}_{h}=u_{0}+v_{h}$. Hence, we can estimate $\tilde{u}_{h}-u$ by estimating $v_{h}-v$. We shall do this by estimating, each individually, $v_{h}-\tilde{v}$ and $\tilde{v}-v$, where $\tilde{v}$ is an appropriate approximation of $v$. In this section we introduce such a $\tilde{v}$ and derive estimates for $\tilde{v}-v$. For the analysis of $v_{h}-\tilde{v}$ we also investigate the regularity of $\tilde{v}$.

Setting

$$
\Phi(x)=\sum_{i, j=1}^{N} \frac{\partial}{\partial x_{j}}\left(\left(a_{i j}(x)-a_{i j}\left(x_{0}\right)\right) \frac{\partial u_{0}}{\partial x_{i}}\right)-\sum_{i=1}^{N} a_{i}(x) \frac{\partial u_{0}}{\partial x_{i}}-a(x) u_{0} \quad \text { in } \Omega,
$$

and

$$
\phi(x)=-\sum_{i, j=1}^{N} a_{i j}(x) \frac{\partial u_{0}}{\partial x_{i}} n_{j}(x) \text { on } \Gamma,
$$

we see from (1.1) and (1.12) that

$$
\begin{array}{ll}
L v=\Phi & \text { in } \Omega, \\
l v=\phi & \text { on } \Gamma .
\end{array}
$$

We shall use the following facts:

LEMMA 4. Let $\Phi, \phi$, and $v$ be defined as above. Then $\phi$ is a smooth function (with degree of smoothness depending on $\operatorname{dist}\left(x_{0}, \Gamma\right)$ ), and the following estimates hold for $\Phi$ and $v($ for $|\beta| \leqslant r$, say $)$ :

$$
\begin{aligned}
& \left|D^{\beta} \Phi(x)\right| \leqslant C\left|x-x_{0}\right|^{-N+1-|\beta|}, \\
& \left|D^{\beta} v(x)\right| \leqslant \begin{cases}C\left(\ln \left(\left|x-x_{0}\right|^{-1}\right)+1\right) & \text { if }-N+3-|\beta|=0, \\
C\left(\left|x-x_{0}\right|^{-N+3-|\beta|}+1\right) & \text { if }-N+3-|\beta| \neq 0 .\end{cases}
\end{aligned}
$$

Proof. The smoothness of $\phi$ and the estimate (3.2) follows at once from the definitions. The estimate (3.3) can be obtained, implicitly, from [8]. For completeness, we show in an appendix that the result follows easily from the properties of the Green's function.

We now introduce $\tilde{v}$, requiring that $\tilde{v}$ be close to $v$, that $\tilde{v} \in H^{2}(\Omega)$, that $\tilde{v}$ possess at least $r$ derivatives away from $x_{0}$, and that $v_{h}$ (which is the Galerkin approximation of $v$ ) be also the Galerkin approximation of $\tilde{v}$. Therefore, let $\tilde{v}$ be the solution of

$$
\begin{array}{ll}
L \tilde{v}=\tilde{\Phi} & \text { in } \Omega, \\
l \tilde{v}=\phi & \text { on } \Gamma,
\end{array}
$$

where $\tilde{\Phi}$ is defined as follows: Set $\varepsilon=h^{1 /(1-\alpha)}$ and let $\Omega_{h}$ be the smallest mesh-domain covering $B_{\varepsilon}\left(x_{0}\right) \cap \Omega, B_{\varepsilon}\left(x_{0}\right) \equiv\left\{x:\left|x-x_{0}\right|<\varepsilon\right\}$. By a mesh-domain we mean 
(the interior of the closure of) a union of elements. Set

$$
\tilde{\Phi}(x)= \begin{cases}\pi_{\tau} \Phi & \text { on } \Omega_{h}, \\ \Phi & \text { outside } \Omega_{h}\end{cases}
$$

where $\pi_{\tau}$ is the local $L_{2}$-projection onto $P_{r-1}(\tau)$, the space of polynomials of degree at most $r-1$ restricted to $\tau$.

It is well known that problem (3.4) admits a (unique) solution, and the following estimates hold for $\tilde{\Phi}$ and $\tilde{v}$ :

Lemma 5. Let $\tilde{\Phi}$ be defined by (3.5) and let $\tilde{v}$ be the solution of (3.4). Then (with $\left.\varepsilon=h^{1 /(1-\alpha)}\right)$

$$
\begin{gathered}
\|\tilde{\Phi}\|_{L_{2}(\Omega)} \leqslant C \varepsilon^{-N / 2+1}(\ln 1 / \varepsilon)^{\bar{N} / 2}, \\
\|\tilde{\Phi}\|_{L_{1}\left(\Omega_{h}\right)} \leqslant C \varepsilon \\
\|\tilde{v}\|_{2, \Omega} \leqslant C \varepsilon^{-N / 2+1}(\ln 1 / \varepsilon)^{\bar{N} / 2}
\end{gathered}
$$

where $\bar{N}=1$ if $N=2, \bar{N}=0$ if $N>2$, and for $j \leqslant J_{1}$ and $r \geqslant 2($ cf. Lemma 1$)$,

$$
\|\tilde{v}\|_{r, D_{j}} \leqslant C d_{j}^{-N / 2+3-r} \text {. }
$$

Proof. We have first

$$
\|\tilde{\Phi}\|_{L_{2}(\Omega)} \leqslant\|\Phi\|_{L_{2}\left(\Omega \backslash \Omega_{h}\right)}+\sum_{\tau \subset \Omega_{h}}\|\tilde{\Phi}\|_{L_{2}(\tau)}
$$

and by (3.2), with $R=\left|x-x_{0}\right|$,

$$
\|\Phi\|_{L_{2}\left(\Omega \backslash \Omega_{h}\right)}^{2} \leqslant C \int_{\varepsilon}^{\operatorname{diam}(\Omega)}\left(R^{-N+1}\right)^{2} R^{N-1} d R \leqslant C \varepsilon^{-N+2}\left(\ln \frac{1}{\varepsilon}\right)^{\bar{N}} .
$$

Using Lemma 3, we obtain

$$
\begin{aligned}
\|\tilde{\Phi}\|_{L_{2}(\tau)}^{2} & =\int_{\tau} \tilde{\Phi} \tilde{\Phi} d x=\int_{\tau} \Phi \tilde{\Phi} d x \leqslant\|\Phi\|_{L_{1}(\tau)}\|\tilde{\Phi}\|_{L_{\infty}(\tau)} \\
& \leqslant C\|\Phi\|_{L_{1}(\tau)} \varepsilon^{-N / 2}\|\tilde{\Phi}\|_{L_{2}(\tau)}
\end{aligned}
$$

and hence, using (3.2),

$$
\sum_{\tau \subset \Omega_{h}}\|\tilde{\Phi}\|_{L_{2}(\tau)} \leqslant C \varepsilon^{-N / 2}\|\Phi\|_{L_{1}\left(\Omega_{h}\right)} \leqslant C \varepsilon^{-N / 2+1}
$$

Together our estimates now prove (3.6).

The estimate (3.7) follows at once from the proof of (3.6), and (3.8) follows from (3.6) by the standard $H^{2}$-regularity estimate, since $\phi$ is smooth (cf., e.g., [9]).

For the proof of (3.9) we note that such an estimate holds for $v$, because of (3.3). Hence,

$$
\|\tilde{v}\|_{r, D_{j}} \leqslant\|\tilde{v}-v\|_{r, D_{j}}+C d_{j}^{-N / 2+3-r}
$$

Further, we have

$$
(\tilde{v}-v)(x)=\int_{\Omega_{h}} g(x, y)(\tilde{\Phi}(y)-\Phi(y)) d y,
$$


where $g$ is the associated Green's function; i.e., $g(x, y)$ is the solution of

$$
A(\psi, g(x, \cdot))=\psi(x) \text { for all } \psi \in W_{\infty}^{1}(\Omega) .
$$

It is known (cf., e.g., [8]) that such a $g$ exists and that

$$
\left|D_{x}^{\beta} D_{y}^{\gamma} g(x, y)\right| \leqslant \begin{cases}C \ln \left(|x-y|^{-1}+1\right) & \text { if }-N+2-|\beta|-|\gamma|=0, \\ C|x-y|^{-N+2-|\beta|-|\gamma|} & \text { if }-N+2-|\beta|-|\gamma|<0 .\end{cases}
$$

Hence, for $x \in D_{j}$ and $|\beta| \leqslant r$,

$$
\left|D^{\beta}(\tilde{v}-v)(x)\right| \leqslant \sup _{y \in \Omega_{h}}\left|D_{x}^{\beta} g(x, y)\right|\left(\|\tilde{\Phi}\|_{L_{1}\left(\Omega_{h}\right)}+\|\Phi\|_{L_{1}\left(\Omega_{h}\right)}\right) \leqslant C d_{j}^{-N+2-r} \varepsilon,
$$

where we have also used (3.2) and (3.7) in the last step. Together our estimates show (3.9) which completes the proof of Lemma 5.

We shall now see that $\tilde{v}$ is appropriately close to $v$.

LEMMA 6. Let $\tilde{v}$ be the solution of (3.4) and $v$ that of (3.1), or, equivalently, set $v=u-u_{0}$. Then

$$
\|\nabla(\tilde{v}-v)\|_{L_{1}(\Omega)} \leqslant C \varepsilon^{2}
$$

and

$$
\|\tilde{v}-v\|_{L_{1}(\Omega)} \leqslant C \varepsilon^{3} \ln 1 / \varepsilon .
$$

Proof. Let $B_{C \varepsilon}\left(x_{0}\right)$ be the ball of smallest radius such that $\Omega_{h} \subset B_{C_{\varepsilon}}\left(x_{0}\right)$ and set $B=B_{2 C \varepsilon}\left(x_{0}\right) \cap \Omega$. We have at once that

$$
\left\|\nabla^{i}(\tilde{v}-v)\right\|_{L_{1}(\Omega)} \leqslant\left\|\nabla^{i}(\tilde{v}-v)\right\|_{L_{1}(B)}+\left\|\nabla^{i}(\tilde{v}-v)\right\|_{L_{1}(\Omega \backslash B)} \quad \text { for } i=0,1,
$$

and, by (3.11) and a change of order of integration,

$$
\begin{aligned}
\left\|\nabla^{i}(\tilde{v}-v)\right\|_{L_{1}(B)} & \leqslant \sup _{y \in \Omega_{h}}\left\|\nabla_{x}^{i} g(\cdot, y)\right\|_{L_{1}(B)}\|\tilde{\Phi}-\Phi\|_{L_{1}\left(\Omega_{h}\right)} \\
& \leqslant C \varepsilon^{2-i}(\ln 1 / \varepsilon)^{\bar{N}(1-i)}\left(\|\tilde{\Phi}\|_{L_{1}\left(\Omega_{h}\right)}+\|\Phi\|_{L_{1}\left(\Omega_{h}\right)}\right) \\
& \leqslant C \varepsilon^{3-i}(\ln 1 / \varepsilon)^{\bar{N}(1-i)} \quad \text { for } i=0,1 .
\end{aligned}
$$

Replacing $g(x, \cdot)$ by its expansion

$$
g(x, y)=g\left(x, x_{0}\right)+\left(y-x_{0}\right) \nabla_{y} g\left(x, x_{0}\right)^{t}+\frac{1}{2}\left(y-x_{0}\right) \nabla_{y}^{2} g(x, \eta)\left(y-x_{0}\right)^{t},
$$

where $\eta=\theta x_{0}+(1-\theta) y, 0 \leqslant \theta \leqslant 1$, and $t$ denotes transpose, and using the fact that $\tilde{\Phi}-\Phi$ is orthogonal on $\Omega_{h}$ to the linear part of the expansion, we see from (3.10) that

$$
(\tilde{v}-v)(x)=\int_{\Omega_{h}}\left(y-x_{0}\right) \nabla_{y}^{2} g(x, \eta)\left(y-x_{0}\right)^{t}(\tilde{\Phi}(y)-\Phi(y)) d y .
$$

Hence, again by (3.11),

$$
\begin{aligned}
\left|\nabla^{i}(\tilde{v}-v)(x)\right| & \leqslant C \varepsilon^{2}\left\|\nabla_{x}^{i} \nabla_{y}^{2} g(x, \cdot)\right\|_{L_{\infty}\left(\Omega_{h}\right)}\left(\|\tilde{\Phi}\|_{L_{1}\left(\Omega_{h}\right)}+\|\Phi\|_{L_{1}\left(\Omega_{h}\right)}\right) \\
& \leqslant C \varepsilon^{3}\left|x-x_{0}\right|^{-N-i} \text { for } x \in \Omega \backslash B, i=0,1 .
\end{aligned}
$$


Integration over $\Omega \backslash B$ shows that

$$
\left\|\nabla^{i}(\tilde{v}-v)\right\|_{L_{1}(\Omega \backslash B)} \leqslant C \varepsilon^{3-i}(\ln 1 / \varepsilon)^{1-i} \text { for } i=0,1 .
$$

This completes the proof of the lemma.

We close this section by noting that also the final claim on $\tilde{v}$ is satisfied, namely that its Galerkin approximation is $v_{h}$. For by (3.1), (3.4), and the definition of $\tilde{\Phi}$, we have

$$
A(\tilde{v}-v, \chi)=(L(\tilde{v}-v), \chi)=(\tilde{\Phi}-\Phi, \chi)=0 \quad \text { for all } \chi \in S_{h},
$$

and by (1.3) and (1.4),

$$
A\left(v_{h}-v, \chi\right)=0 \quad \text { for all } \chi \in S_{h},
$$

so that

$$
A\left(v_{h}-\tilde{v}, \chi\right)=0 \quad \text { for all } \chi \in S_{h} .
$$

4. Proof of Theorem 1. In view of Lemma 6 and the fact that $\tilde{u}_{h}-u=v_{h}-v$, it is sufficient to show that for the appropriate $\alpha$ 's

$$
\left\|\nabla\left(v_{h}-\tilde{v}\right)\right\|_{L_{1}(\Omega)} \leqslant C h^{r-1},
$$

and

$$
\left\|v_{h}-\tilde{v}\right\|_{L_{1}(\Omega)} \leqslant C h^{r}
$$

respectively.

Given a positive constant $C_{*}$ let $J$ be determined by

$$
C_{*} h^{1 /(1-\alpha)} \leqslant d_{J}<2 C_{*} h^{1 /(1-\alpha)} \text {. }
$$

Thus, $h_{J}, d_{J}$, and $h^{1 / 1-\alpha)}$ are of the same order; but, by choosing $C_{*}$ sufficiently large, $h_{J} d_{J}^{-1}$ is suitably small, since

$$
h_{J} d_{J}^{-1}=h d_{J}^{\alpha-1} \leqslant 1 / C_{*}^{1-\alpha} .
$$

The constant $C_{*}$ will be determined later. For the moment we only require that the results of Lemma 1, Lemma 2 , and (3.9) apply for $j \leqslant J$, i.e., that $C_{*} \geqslant C_{1}$.

In order to prove (4.1) we first use Schwarz's inequality to change from the $L_{1}$-norm to a weighted $L_{2}$-norm. Setting $e=v_{h}-\tilde{v}$ and

$$
S=\sum_{j \leqslant J} d_{j}^{N / 2}\|e\|_{1, D_{j}}
$$

we have

$$
\|\nabla e\|_{L_{1}(\Omega)}=\sum_{j \leqslant J}\|\nabla e\|_{L_{1}\left(D_{j}\right)}+\|\nabla e\|_{L_{1}\left(\Omega_{J+1}\right)} \leqslant C S+d_{J}^{N / 2}\|e\|_{1, \Omega_{J+1}} .
$$

We shall show that for $\alpha>(r-3) /(r-1)$ and a suitable choice of $C_{*}$,

$$
S \leqslant \frac{1}{2} S+C d_{J}^{N / 2}\|e\|_{1, \Omega,}+C h^{r-1}
$$

and

$$
d_{J}^{N / 2}\|e\|_{1, \Omega} \leqslant C h^{r-1} .
$$

Obviously, the desired result then follows. 
By Lemma 2, we have

$$
\begin{aligned}
S & \leqslant C \sum_{j<J} d_{j}^{N / 2}\left(h_{j}^{r-1}\|\tilde{v}\|_{r, D_{j}^{1}}+d_{j}^{-1}\|e\|_{0, D_{j}^{1}}\right)+d_{J}^{N / 2}\|e\|_{1, D_{J}} \\
& \leqslant C \sum_{j \leqslant J} d_{j}^{N / 2} h_{j}^{r-1}\|\tilde{v}\|_{r, D_{j}}+C \sum_{j \leqslant J} d_{j}^{N / 2-1}\|e\|_{0, D_{j}}+d_{J}^{N / 2}\|e\|_{1, D_{J}},
\end{aligned}
$$

and by Lemma 5 and our assumption $\alpha>(r-3) /(r-1)$,

$$
\begin{aligned}
\sum_{j \leqslant J} d_{j}^{N / 2} h_{j}^{r-1}\|\tilde{v}\|_{r, D_{j}} & \leqslant C \sum_{j_{1} \leqslant j \leqslant J} h_{j}^{r-1} d_{j}^{3-r} \\
& \leqslant C h^{r-1} \sum_{j_{1} \leqslant j} d_{j}^{\alpha(r-1)+3-r} \leqslant C h^{r-1} .
\end{aligned}
$$

In order to estimate $\|e\|_{0, D_{j}}$ we use duality. Let $e_{j}$ equal $e /\|e\|_{0, D_{j}}$ on $D_{j}$ and vanish outside $D_{j}$, and let $w$ solve

$$
A(\psi, w)=\left(\psi, e_{j}\right) \text { for all } \psi \in H^{1}(\Omega) .
$$

Hence, $\|e\|_{0, D_{j}}=\left(e, e_{j}\right)=A(e, w)$, and by (3.14) and Lemma 1,

$$
\|e\|_{0, D_{j}}=A\left(e, w-w_{I}\right) \leqslant C \sum_{i \leqslant J}\|e\|_{1, D_{i}} h_{i}\|w\|_{2, D_{i}^{1}}+C\|e\|_{1, \Omega_{J+1}} h_{J}\|w\|_{2, \Omega_{J}} \text {. }
$$

It is well known that problem (4.8) admits a unique solution $w$ such that

$$
\|w\|_{2, \Omega} \leqslant C\left\|e_{j}\right\|_{0, \Omega}
$$

and with the representation

$$
w(x)=\int_{\Omega} g^{*}(x, y) e_{j}(y) d y,
$$

with a $g^{*}$ (the Green's function for the adjoint problem) such that

$$
\left|D_{x}^{\beta} g^{*}(x, y)\right| \leqslant C|x-y|^{-N} \text { for }|\beta| \leqslant 2 .
$$

Hence, for $w$ we have the estimates

$$
\begin{array}{ll}
\|w\|_{2, D_{i}} \leqslant C d_{i}^{-N / 2} d_{j}^{N / 2} & \text { for } i \leqslant j, \\
\|w\|_{2, \Omega_{i}} \leqslant C d_{i}^{N / 2} d_{j}^{-N / 2} & \text { for } i \geqslant j .
\end{array}
$$

For $i=j-1, j$, and $j+1$ this follows from (4.9), since $e_{j}$ has $L_{2}$-norm equal to one, and for the other $i$ 's from the representation (4.10) and the estimate (4.11). We have thus the following estimate:

$$
\begin{aligned}
\|e\|_{0, D_{j}} \leqslant & C d_{j}^{N / 2} \sum_{i \leqslant j}\|e\|_{1, D_{i}} h_{i} d_{i}^{-N / 2}+C d_{j}^{-N / 2} \sum_{j<i \leqslant J}\|e\|_{1, D_{i}} h_{i} d_{i}^{N / 2} \\
& +C\|e\|_{1, \Omega_{j+1}} h_{J} d_{J}^{N / 2} d_{j}^{-N / 2} .
\end{aligned}
$$

Using obvious arguments, we obtain

$$
\begin{aligned}
\|e\|_{0, D_{j}} \leqslant & C d_{j}^{N / 2} \max _{i \leqslant j}\left(h_{i} d_{i}^{-N}\right) \sum_{i \leqslant j}\|e\|_{1, D_{i}} d_{i}^{N / 2} \\
& +C h_{j} d_{j}^{-N / 2} \sum_{j<i \leqslant J}\|e\|_{1, D_{i}} d_{i}^{N / 2}+C\|e\|_{1, \Omega_{J+1}} h_{J} d_{J}^{N / 2} d_{j}^{-N / 2} \\
\leqslant & C h_{j} d_{j}^{-N / 2} S+C\|e\|_{1, \Omega_{J+1}} h_{J} d_{J}^{N / 2} d_{j}^{-N / 2}
\end{aligned}
$$


and hence, using (4.3),

$C \sum_{j \leqslant J} d_{j}^{N / 2-1}\|e\|_{0, D_{j}} \leqslant C S \sum_{j \leqslant J} h_{j} d_{j}^{-1}+C\|e\|_{1, \Omega_{J+1}} h_{J} d_{J}^{N / 2} \sum_{j \leqslant J} d_{j}^{-1}$
$\leqslant C S h_{J} d_{J}^{-1}+C\|e\|_{1, \Omega_{J+1}} h_{J} d_{J}^{N / 2} d_{J}^{-1} \leqslant\left(C / C_{*}^{1-\alpha}\right) S+C d_{J}^{N / 2}\|e\|_{1, \Omega_{J+1}}$.

For a suitable choice of $C_{*}$ and together with (4.6) and (4.7) this shows (4.4).

We now prove (4.5). By the coercivity and the continuity of $A(\cdot, \cdot)$, and by (3.14), we have

$$
\|e\|_{1, \Omega}^{2} \leqslant C A(e, e)=C A(e, \tilde{v}-\chi) \leqslant C\|\tilde{v}-\chi\|_{1, \Omega}^{2} \quad \text { for all } \chi \in S_{h} .
$$

Lemma 1 then shows

$$
\|e\|_{1, \Omega}^{2} \leqslant C \sum_{j<J} h_{j}^{2(r-1)}\|\tilde{v}\|_{r, D_{j}^{1}}^{2}+C h_{J}^{2}\|\tilde{v}\|_{2, \Omega_{J-1}}^{2},
$$

and by Lemma 5 ,

(4.13) $\|e\|_{1, \Omega}^{2} \leqslant C \sum_{j_{1} \leqslant j \leqslant J} h_{j}^{2(r-1)} d_{j}^{2(-N / 2+3-r)}+C h_{J}^{2} h^{(-N+2) /(1-\alpha)}\left(\ln \frac{1}{h}\right)^{\bar{N}}$,

since $\varepsilon=h^{1 /(1-\alpha)}$. But for $\alpha>(r-3) /(r-1)$,

$$
\sum_{j_{1} \leqslant j \leqslant J} h_{j}^{2(r-1)} d_{j}^{2(-N / 2+3-r)} \leqslant d_{J}^{-N} h^{2(r-1)} \sum_{j_{1} \leqslant j} d_{j}^{2 \alpha(r-1)+2(3-r)} \leqslant C d_{J}^{-N} h^{2(r-1)},
$$

and

$$
h_{J}^{2} h^{(-N+2) /(1-\alpha)}(\ln 1 / h)^{\bar{N}} \leqslant C\left(C_{*}\right) d_{J}^{-N} h^{4 /(1-\alpha)}(\ln 1 / h)^{\bar{N}} \leqslant C\left(C_{*}\right) d_{J}^{-N} h^{2(r-1)} .
$$

Together these estimates show (4.5) which completes the proof of (4.1).

We now turn to the proof of (4.2). We have at once

$$
\|e\|_{L_{1}(\Omega)} \leqslant C \sum_{j \leqslant J} d_{j}^{N / 2}\|e\|_{0, D_{j}}+d_{J}^{N / 2}\|e\|_{0, \Omega_{J+1}} .
$$

Applying (4.12) and changing order of summation we obtain

$$
\begin{aligned}
\sum_{j \leqslant J} d_{j}^{N / 2}\|e\|_{0, D_{j}} \leqslant & C \sum_{i \leqslant J}\|e\|_{1, D_{i}} h_{i} d_{i}^{-N / 2} \sum_{i \leqslant j \leqslant J} d_{j}^{N} \\
& +C \sum_{i \leqslant J}\|e\|_{1, D_{i}} h_{i} d_{i}^{N / 2} \sum_{j_{1} \leqslant j<i} 1+C\|e\|_{1, \Omega_{J+1}} h_{J} d_{J}^{N / 2} \sum_{j_{1} \leqslant j \leqslant J} 1,
\end{aligned}
$$

and hence (for convenience we now assume that $j_{1} \geqslant 1$ ),

$$
\sum_{j \leqslant J} d_{j}^{N / 2}\|e\|_{0, D_{j}} \leqslant C \sum_{i \leqslant J} i h_{i} d_{i}^{N / 2}\|e\|_{1, D_{i}}+C J h_{J} d_{J}^{N / 2}\|e\|_{1, \Omega_{J+1}} .
$$

We shall show that the single term in (4.14) can be estimated in the same way. Repeating the arguments used to derive (4.12), we obtain

$$
\|e\|_{0, \Omega_{J+1}} \leqslant C \sum_{i \leqslant J}\|e\|_{1, D_{i}} h_{i}\|w\|_{2, D_{1}^{1}}+C\|e\|_{1, \Omega_{J+1}} h_{J}\|w\|_{2, \Omega_{J}}
$$

where $w$ now is the solution of the problem

$$
A(\psi, w)=\left(\psi, e_{J+1}\right) \quad \text { for all } \psi \in H^{1}(\Omega),
$$

for an appropriate $e_{J+1}$ with $L_{2}$-norm equal to one. Hence by (4.9),

$$
d_{J}^{N / 2}\|e\|_{0, \Omega_{J+1}} \leqslant C d_{J}^{N / 2}\left(\sum_{i \leqslant J} h_{i}\|e\|_{1, D_{i}}+h_{J}\|e\|_{1, \Omega_{J+1}}\right)
$$


which is an even better estimate than we required. We have thus shown that

$$
\|e\|_{L_{1}(\Omega)} \leqslant C \sum_{j \leqslant J} j h_{j} d_{j}^{N / 2}\|e\|_{1, D_{j}}+C J h_{J} d_{J}^{N / 2}\|e\|_{1, \Omega_{J+1}} .
$$

Now, set

$$
S^{\prime}=\sum_{j \leqslant J} j h_{j} d_{j}^{N / 2}\|e\|_{1, D_{j}}
$$

We shall show that for $\alpha>(r-3) / r$ and a sufficiently large $C_{*}$,

$$
S^{\prime} \leqslant \frac{1}{2} S^{\prime}+C J h_{J} d_{J}^{N / 2}\|e\|_{1, \Omega_{J}}+C h^{r}
$$

and

$$
J h_{J} d_{J}^{N / 2}\|e\|_{1, \Omega} \leqslant C h^{r} .
$$

Clearly the desired result then follows.

By Lemma 2,

$$
S^{\prime} \leqslant C \sum_{j \leqslant J} j h_{j}^{r} d_{j}^{N / 2}\|\tilde{v}\|_{r, D_{j}}+C \sum_{j \leqslant J} j h_{j} d_{j}^{N / 2-1}\|e\|_{0, D_{j}}+J h_{J} d_{J}^{N / 2}\|e\|_{1, \Omega_{j}},
$$

and by Lemma 5 and our assumption $\alpha>(r-3) / r$,

$$
\sum_{j \leqslant J} j h_{j}^{r} d_{j}^{N / 2}\|\tilde{v}\|_{r, D_{j}} \leqslant C h^{r} \sum_{j_{1} \leqslant j \leqslant J} j d_{j}^{r \alpha+3-r} \leqslant C h^{r} .
$$

Using (4.12), we have

$$
\begin{aligned}
\sum_{j \leqslant J} j h_{j} d_{j}^{N / 2-1}\|e\|_{0, D_{j}} \leqslant & C \sum_{i \leqslant J}\|e\|_{1, D_{i}} h_{i} d_{i}^{-N / 2} \sum_{i \leqslant j \leqslant J} j h_{j} d_{j}^{N-1} \\
& +C \sum_{i \leqslant J}\|e\|_{1, D_{i}} h_{i} d_{i}^{N / 2} \sum_{j_{1} \leqslant j<i} j h_{j} d_{j}^{-1} \\
& +C\|e\|_{1, \Omega_{J+1}} h_{J} d_{J}^{N / 2} \sum_{j_{1} \leqslant j \leqslant J} j h_{j} d_{j}^{-1}
\end{aligned}
$$

which shows that

$$
\sum_{j \leqslant J} j h_{j} d_{j}^{N / 2-1}\|e\|_{0, D_{j}} \leqslant C \sum_{i \leqslant J}\|e\|_{1, D_{i}} i h_{i}^{2} d_{i}^{N / 2-1}+C\|e\|_{1, \Omega_{J+1}} J h_{J}^{2} d_{J}^{N / 2-1},
$$

and, hence, that

$$
C \sum_{j \leqslant J} j h_{j} d_{j}^{N / 2-1}\|e\|_{0, D_{j}} \leqslant\left(C / C_{*}^{1-\alpha}\right) S^{\prime}+C J h_{J} d_{J}^{N / 2}\|e\|_{1, \Omega_{J+1}} .
$$

For $C_{*}$ sufficiently large our estimates now together show (4.15).

It remains to prove (4.16). Since for $j \leqslant J$

$$
J h_{J} d_{J}^{N / 2} \leqslant j h_{j} d_{j}^{N / 2}
$$

we obtain from (4.13) that

$$
J^{2} h_{J}^{2} d_{J}^{N}\|e\|_{1, \Omega}^{2} \leqslant C \sum_{j_{1} \leqslant j \leqslant J} j^{2} h_{j}^{2 r} d_{j}^{2(3-r)}+C J^{2} h_{J}^{4} d_{J}^{N} h^{(-N+2) /(1-\alpha)}\left(\ln \frac{1}{h}\right)^{\bar{N}}
$$


so for $\alpha>(r-3) / r$,

$$
J^{2} h_{J}^{2} d_{J}^{N}\|e\|_{1, \Omega}^{2} \leqslant C h^{2 r} \sum_{j_{1} \leqslant j} j^{2} d_{j}^{2 \alpha r+2(3-r)}+C\left(C_{*}\right) J^{2} h^{6 /(1-\alpha)}\left(\ln \frac{1}{h}\right)^{\bar{N}} \leqslant C h^{2 r} .
$$

This completes the proof of (4.2) and hence of Theorem 1.

5. Proof of Theorem 2. We shall show that for $\alpha>(r-3) / r$,

$$
\left|\tilde{u}_{h}(x)-u(x)\right| \leqslant C h^{r}(\ln 1 / h)^{\bar{r}}\left|x-x_{0}\right|^{-N} .
$$

In view of Theorem 1 and the fact that $\tilde{u}_{h}-u$ equals $v_{h}-v$, it is sufficient to show that

$$
\left|v_{h}(x)-v(x)\right| \leqslant C h^{r}(\ln 1 / h)^{\bar{r}} d^{-N}+C d^{-N}\left\|v_{h}-v\right\|_{L_{1}(\Omega)},
$$

where $d=\left|x-x_{0}\right|$.

We first consider the case when $d>c h^{1 /(1-\alpha)}$ and $c$ is a sufficiently large constant. Let $B$ be the intersection of $\Omega$ and $B_{d / 2}(x)$, i.e., set

$$
B=\left\{x \in \Omega:\left|x-x_{0}\right|<\frac{1}{2} d\right\} .
$$

Following the arguments in [10] (cf. Corollary 5.1 of [10] and the remark below), we deduce that

$$
\left|v_{h}(x)-v(x)\right| \leqslant C\left(h d^{\alpha}\right)^{r}\left(\ln \frac{1}{h}\right)^{\bar{r}} \max _{|\beta| \leqslant r}\left\|D^{\beta} v\right\|_{L_{\infty}(B)}+C d^{-N}\left\|v_{h}-v\right\|_{L_{1}(B)} .
$$

Hence (5.1) follows from (3.3) and our assumption $\alpha>(r-3) / r$.

In the case $d \leqslant c h^{1 /(1-\alpha)}$ we first use Lemma 3. Thinking of $v(x)$ as a constant on $\bar{\tau} \ni x$ we obtain

$$
\begin{aligned}
\left|v_{h}(x)-v(x)\right| & \leqslant C h^{-N /(1-\alpha)}\left\|v_{h}-v(x)\right\|_{L_{1}(\tau)} \\
& \leqslant C h^{-N /(1-\alpha)}\left(\left\|v_{h}-v\right\|_{L_{1}(\tau)}+\|v-v(x)\|_{L_{1}(\tau)}\right) .
\end{aligned}
$$

It remains to show that

$$
\|v-v(x)\|_{L_{1}(\tau)} \leqslant C h^{N /(1-\alpha)} h^{r} d^{-N} .
$$

For $N>2$ this follows at once from (3.3), since

$$
\|v\|_{L_{1}(\tau)}+h^{N /(1-\alpha)}|v(x)| \leqslant C h^{N /(1-\alpha)} d^{-N} h^{r} .
$$

For $N=2$ we first note that for $y \in \tau$ and a suitable curve $S$ we have, using (3.3),

$$
|v(y)-v(x)|=\left|\int_{S} v^{\prime}(s) d s\right| \leqslant C h^{1 /(1-\alpha)} \max \left(\ln \frac{1}{d}, \ln \frac{1}{\left|y-x_{0}\right|}\right),
$$

and hence

$$
\|v-v(x)\|_{L_{1}(\tau)} \leqslant C h^{3 /(1-\alpha)} \ln 1 / d \leqslant C h^{2 /(1-\alpha)} h^{r} d^{-2} .
$$

This completes the proof.

Remark. For $x$ bounded away from $\Gamma$ the local estimate (5.2) follows at once from Corollary 5.1 in [10]. Following the arguments in [10] and using cut-off functions which satisfy an appropriate boundary condition (the vanishing of the conormal 
derivative on $\Gamma$ ), it is easy to see that (5.2) holds also in the general case. Such cut-off functions were also used in [6].

\section{Appendix.}

Proof of (3.3): Recall that by definition $v=u-u_{0}, L v=\Phi$, and $l v=\phi$. We shall show that for, say, $|\beta| \leqslant r$

$$
\left|D^{\beta} v(x)\right| \leqslant \begin{cases}C\left(\left|x-x_{0}\right|^{-N+3-|\beta|}+1\right) & \text { if }|\beta| \neq 3-N \\ C \ln \left(\left|x-x_{0}\right|^{-1}+1\right) & \text { if }|\beta|=3-N\end{cases}
$$

Let $x \neq x_{0}$ be given and set $d=\left|x-x_{0}\right|$. Since $v=u-u_{0}$, and both $u$ and $u_{0}$ are smooth functions away from $x_{0}$, it is sufficient to consider the case when $d$ is suitably small. Let $\omega$ be a smooth cut-off function such that

$$
\omega=1 \quad \text { in } A_{1}, \quad \operatorname{supp}(\omega) \subset \overline{A_{2}}, \quad \text { and }\left\|D^{\gamma} \omega\right\|_{L_{\infty}(\Omega)} \leqslant C d^{-|\gamma|},
$$

where $A_{i}=\left\{y \in \Omega:(i+1)^{-1} d<\left|y-x_{0}\right|<(i+1) d\right\}$. Let $g(y, z)$ be the Green's function for $L$ and $l$; i.e., let $g$ be the solution of $L_{z}^{*} g(y, z)=\delta(z-y)$ in $\Omega$, $l_{z}^{*} g(y, z)=0$ on $\Gamma$. Using Green's formula and a splitting of $\Phi$ we can write $v$ as the sum of three terms

$$
v=\int_{\Omega} g \omega \Phi d x+\int_{\Omega} g(1-\omega) \Phi d z+\int_{\Gamma} g \phi d \Gamma(z)=v_{1}+v_{2}+v_{3},
$$

where the latter identity defines $v_{1}, v_{2}$, and $v_{3}$. For $d$ sufficiently small, $x$ is bounded away both from $\Gamma$ and the support of $(1-\omega) \Phi$, and thus, in a neighborhood of $x$, we can differentiate $v_{2}$ and $v_{3}$ under the integral signs. Using (3.2), (3.11), and straightic.ward calculations, we obtain

$$
\begin{aligned}
\left|D^{\beta} v_{2}(x)\right| & \leqslant \int_{\Omega}\left|D^{\beta} g(x, z)\right||(1-\omega(z)) \Phi(z)| d z \\
& \leqslant C \int_{\Omega \backslash A_{1}} \max \left(\ln |x-z|^{-1},|x-z|^{-N+2-|\beta|}\right)\left|z-x_{0}\right|^{-N+1} d z \\
& \leqslant \begin{cases}C\left(d^{-N+3-|\beta|}+1\right) & \text { if }|\beta| \neq 3-N, \\
C \ln 1 / d & \text { if }|\beta|=3-N,\end{cases}
\end{aligned}
$$

and

$$
\left|D^{\beta} v_{3}(x)\right| \leqslant \int_{\Gamma}\left|D^{\beta} g(x, z)\right||\phi(z)| d \Gamma(z) \leqslant C .
$$

Similarly, we obtain for $y \in \Omega \backslash A_{3}$,

$$
\left|D^{\gamma} v_{1}(y)\right| \leqslant \begin{cases}C d^{-N+3-|\gamma|}(\ln 1 / d)^{\mu} & \text { if }\left|y-x_{0}\right|<d / 4 \\ C d\left|y-x_{0}\right|^{-N+2-|\gamma|}\left\{\ln \left(\left|y-x_{0}\right|^{-1}+1\right)\right\}^{u} & \text { if }\left|y-x_{0}\right|>4 d\end{cases}
$$

where $\mu=1$ if $|\gamma|=2-N$ and $\mu=0$ otherwise. In order to estimate $D^{\beta} v_{1}(x)$ we proceed as follows: For $|\beta| \leqslant 1$ we have at once that

$$
\begin{aligned}
\left|D^{\beta} v_{1}(x)\right| & \leqslant\left\|D^{\beta} g(x, \cdot)\right\|_{L_{1}\left(A_{2}\right)}\|\omega \Phi\|_{L_{\infty}\left(A_{2}\right)} \\
& \leqslant C d^{2-|\beta|}(\ln 1 / d)^{\bar{N}(1-|\beta|)} d^{-N+1} \leqslant C\left(d^{-N+3-|\beta|}+1\right) .
\end{aligned}
$$


For $|\beta|>1$, set $D^{\beta}=D^{\sigma} D^{\mu}$ and $w=D^{\mu} v_{1}$ for some $\sigma$ and $\mu$ such that $|\sigma|=1$. Since $L w=D^{\mu}(\omega \Phi)+\eta$ for some $\eta=\sum_{|\gamma| \leqslant|\beta|} b_{\gamma} D^{\gamma} v_{1}$, where $b_{\gamma}$ are certain derivatives of the coefficients of $L$, we have

$$
w=\int_{\Omega} g D^{\mu}(\omega \Phi) d x+\int_{\Omega} g \eta d z+\int_{\Gamma} g l w d \Gamma(z) \equiv w_{1}+w_{2}+w_{3} .
$$

Here

$$
\left|D^{\sigma} w_{1}(x)\right| \leqslant\left\|D_{x}^{\sigma} g(x, \cdot)\right\|_{L_{1}\left(A_{2}\right)}\left\|D^{\mu}(\omega \Phi)\right\|_{L_{\infty}\left(A_{2}\right)} \leqslant C d d^{-N+1-|\mu|}=C d^{-N+3-|\beta|},
$$

and

$$
\left|D^{\sigma} w_{3}(x)\right| \leqslant \int_{\Gamma}\left|D_{x}^{\sigma} g(x, z)\right||l w(z)| d \Gamma(z) \leqslant C,
$$

since $l w$ is smooth. In order to estimate $D^{\sigma} w_{2}(x)$ we first use (6.1) to obtain

$$
\left|\int_{\Omega \backslash A_{3}} D_{x}^{\sigma} g(x, z) \eta(z) d z\right| \leqslant C d^{-N+4-|\beta|},
$$

and hence

$$
\left|D^{\sigma} w_{2}(x)\right| \leqslant C d^{-N+4-|\beta|}+C d \sup _{\substack{|\gamma| \leqslant|\beta| \\ y \in A_{3}}}\left|D^{\gamma} v_{1}(y)\right| .
$$

We have thus shown

$$
\left|D^{\beta} v_{1}(x)\right|=\left|D^{\sigma} w(x)\right| \leqslant C d^{-N+3-|\beta|}+C d \sup _{\substack{\gamma|\leqslant| \beta \mid \\ y \in A_{3}}}\left|D^{\gamma} v_{1}(y)\right|
$$

Since we may as well assume that the supremum is attained for $\gamma=\beta$ and $y=x$, and since $d$ is small, it follows that

$$
\left|D^{\beta} v_{1}(x)\right| \leqslant C d^{-N+3-|\beta|} .
$$

This completes the proof.

Department of Mathematics

Chalmers University of Technology and

The University of Göteborg

S-412 96 Göteborg, Sweden

1. I. BABUSKA, "Error bounds for the finite element method," Numer. Math., v. 16, 1971, pp. 322-333.

2. I. BABUSKA \& A. K. AzIZ, "Survey lectures on the mathematical foundations of the finite element method," Section 6.3.6 of The Mathematical Foundations of the Finite Element Method with Applications to Partial Differential Equations, (A. K. Aziz, ed.), Academic Press, New York, 1972.

3. J. H. BRAmble \& A. H. Schatz, "Estimates for spline projections," RAIRO Anal. Numér., v. 10, 1976, pp. 5-37.

4. P. G. Ciarlet, The Finite Element Method for Elliptic Problems, North-Holland, Amsterdam, 1978.

5. P. G. Ciarlet, “Discrete variational Green's function. I,” Aequationes Math., v. 4, 1970, pp. 74-82.

6. K. ERIKSson, Improved Convergence by Mesh-Refinement in the Finite Element Method, Thesis, Chalmers University of Technology and the University of Göteborg, 1981. 
7. K. ERIKSSON, "Improved accuracy by adapted mesh-refinements in the finite element method," Math. Comp. (this issue).

8. JU. P. KRASOvSKII, "Isolation of singularities of the Green's function," Math. USSR-Izv., v. 1, 1967, pp. 935-966.

9. J. L. Lions \& E. Magenes, Problèmes aux Limites Non Homogènes et Applications, Vol. 1, Dunod, Paris, 1968.

10. A. H. Schatz \& L. B. Wahlbin, "Interior maximum norm estimates for finite element methods," Math. Comp., v. 31, 1977, pp. 414-442.

11. R. Scotr, "Finite element convergence for singular data," Numer. Math., v. 21, 1973, pp. 317-327. 